\title{
On the Performance of a Wireless Powered Communication System Using a Helping Relay
}

\author{
Tan N. NGUYEN ${ }^{1}$, Phuong T. TRAN ${ }^{1}$, Hoang-Sy NGUYEN ${ }^{1,2}$, \\ Dinh-Thuan DO ${ }^{3}$, Miroslav VOZNAK ${ }^{1,2}$
${ }^{1}$ Wireless Communications Research Group, Faculty of Electrical and Electronics Engineering, Ton Duc Thang University, No. 19 Nguyen Huu Tho Street, Tan Phong Ward, District 7, Ho Chi Minh City, Vietnam
2 VSB Technical University of Ostrava, 17. listopadu 15/2172, 70833 Ostrava - Poruba, Czech Republic
${ }^{3}$ Faculty of Electronics Technology, Industrial University of Ho Chi Minh City, Vietnam

\{nguyennhattan, tranthanhphuong, nguyenhoangsy\}@tdt.edu.vn, dodinhthuan@iuh.edu.vn, miroslav.voznak@vsb.cz

Submitted December 16, 2016 / Accepted June 9, 2017

\begin{abstract}
This paper studies the outage performance and system throughput of a bidirectional wireless information and power transfer system with a helping relay. The relay helps forward wireless power from the access point (AP) to the user, and also the information from the user to the AP in the reverse direction. We assume that the relay uses time switching based energy harvesting protocol. The analytical results provide theoretical insights into the effect of various system parameters, such as time switching factor, source transmission rate, transmitting-power-to-noise ratio to system performance for both amplify-and-forward and decode-and-forward relaying protocols. The optimal time switching ratio is determined in each case to maximize the information throughput from the user to the AP subject to the energy harvesting and consumption balance constraints at both the relay and the user. All of the above analyses are confirmed by Monte-Carlo simulation.
\end{abstract}

\section{Keywords}

Amplify-and-forward, bidirectional relay, decode-andforward, wireless powered communications, timeswitching

\section{Introduction}

Recently, radio frequency (RF) signal based wireless energy transfer (WET) has emerged as a perpetual and costeffective solution to power wireless devices, such as mobile sensors, electronic tags, etc. [1]. While numerous works have focused on WET systems to optimize the energy harvesting process at energy receivers [2], the authors of this paper are more interested in another line of WET research, where WET could be integrated with wireless communication by exploiting the dual use of RF signals. Especially, we focus on wireless powered communication (WPC) [3], where the energy for wireless communication at the device is obtained via the WET technology. This advanced technology has been deployed and investigated in various wireless system models, including cellular networks [4], relay systems [5], [6], cognitive radio networks [7], [8].

In last decade, wireless sensor networks have been more and more attracted by research community, due to their ability to carry out different kinds of tasks, from traffic monitoring, agriculture monitoring, to smart home and health-care applications. For these networks, network life time is a critical aspect to the success of the system. By some previous research, battery life time is the bottleneck in determining the life time of the whole system. In [9], three wireless powered sensor network models for infrastructure monitoring application have been proposed and their performances have been investigated. Another wireless powered sensor network model was presented in [10], in which a number of sensor nodes send common information to a far apart information access point via distributed beamforming, by using the wireless energy transferred from a set of nearby multi-antenna energy transmitters.

Both of the works in [9] and [10] only introduce normal sensor networks without the helping of relay nodes. Furthermore, the RF energy transmitters in those works are independent of the information transfer process. This would increase the cost of implementation of these models in practice. In [11], the authors have tried to overcome this drawback by considering a new WPC system, where a wireless user communicates with an access point (AP) assisted by a bidirectional relay. The user and the relay are both powered by the RF energy from the AP. Here, the role of the relay is to forward the energy from the AP to the user, as well as to forward information from the user to the AP. However, the authors in [11] only considered the case that channel gains are constant, and estimate the maximum achievable throughput of the system. Because of this limitation, there is a large difficulty to apply this result to practical sensor networks. In addition, the work in [11] only considers amplify-andforward as the relaying strategy. 
Continuing to the work of [11], in this paper we provide a rigorous analysis on the same wireless powered sensor network model. We apply a Rayleigh distribution model for the channel gains between nodes, including the AP, relay node and the wireless user. For information transfer, both amplify-and-forward (AF) and decode-and-forward (DF) relaying protocols are investigated. Regarding to the energy harvesting protocol, we focus on time switching (TS) strategy at the relay. The outage probability and the average throughput of the system are derived mathematically. The optimal time switching factor to maximize the system throughput is obtained via numerical algorithm. To verify the analysis mentioned above, Monte-Carlo simulations are also conducted and the results are reported in this paper, too.

The rest of this paper is organized as follows. The next section introduces the system model that we are going to analyze. The detailed performance analysis is provided in Sec. 3. The numerical results to support the analysis are given in Sec. 4. Finally, Sec. 5 concludes the paper.

\section{System Model}

We consider a wireless powered system as illustrated in Fig. 1, where a mobile user is intended to send information to the AP with the assistance of a relay R. Assume that both the user and relay $\mathrm{R}$ have no other energy supply but solely the energy harvested from the AP. Furthermore, we assume that direct connection between the AP and the user is so weak, hence, the only available communication path as well as power transfer path is via the relay $\mathrm{R}$. The relay serves the dual roles of both energy relaying from the AP to the user and information forwarding from the user to the AP [11]. To initialize the communication process, a sufficient amount of initial energy is stored in the battery to conduct the first transmission block before energy harvesting, as in [12]. After that, the energy consumed by the user/relay is kept lower than or at most equal to the harvested energy amount during each block, thus no further manual battery replacement/recharging is needed.

All nodes are assumed to operate in half-duplex mode, and either amplify-and-forward (AF) or decode-and-forward (DF) relaying strategy can be used at the relay for information transferring. Regarding to the channel model, we consider the case that perfect channel state information (CSI)

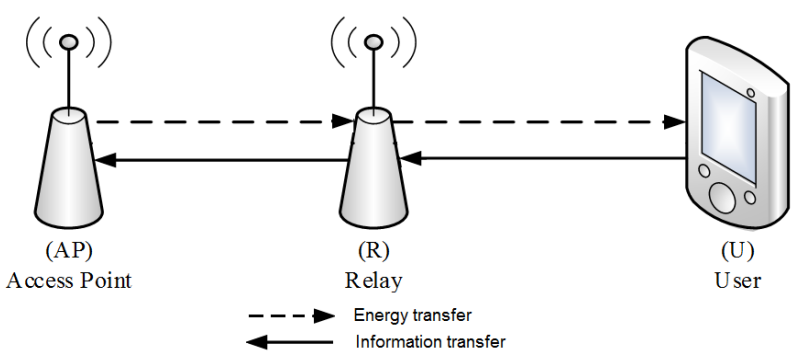

Fig. 1. System model.

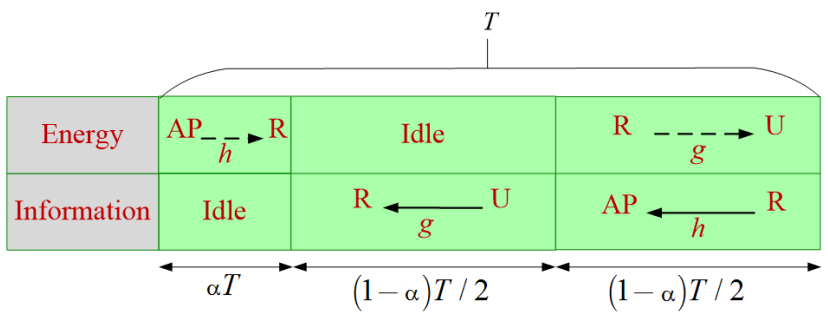

Fig. 2. Time switching based protocol.

is available at the relay and the AP. Let $h$ and $g$ denote the channels from the AP to the relay and from the user to the relay, respectively. In addition, we assume for simplicity that these channels are reciprocal. Different from the work in [11], all channels here experience Rayleigh fading and keep constant during each transmission block so that they can be considered as slow fading. As a result, $|h|^{2}$ and $|g|^{2}$ are an exponential random variables with parameters $\lambda_{h}$ and $\lambda_{g}$, respectively.

For energy harvesting, we employ the time switching relaying (TSR) protocol, which is more convenient to implement in practice. As shown in Fig. 2, the total symbol duration $\mathrm{T}$ is divided into three intervals with the lengths of $\alpha T,(1-\alpha) T / 2$, and $(1-\alpha) T / 2$, respectively, where $0<\alpha<1$ denotes the time-switching ratio. The first interval corresponds to the energy harvesting phase at the relay $\mathrm{R}$, in which the AP wirelessly sends its energy to $\mathrm{R}$ with power $P_{\text {ap }}$. Then, the total energy harvested at R during each block is given by $E_{\mathrm{r}}=\eta P_{\mathrm{ap}} \cdot|h|^{2} \cdot \alpha T$, where $0 \leq \eta \leq 1$ is the energy conversion efficiency. The second phase of duration $(1-\alpha) T / 2$ corresponds to the information transmission from the user to the relay. In the third phase of the transmission block, $\mathrm{R}$ forwards an amplified or decoded signal to the AP and also forwards energy to the user. We assume that the circuit power consumption is negligible as compared to the radiation power, which is reasonable for low-power devices such as sensor nodes.

\section{Performance Analysis}

In this section, the throughput and outage performance of the proposed system are analyzed mathematically. The impact of time-switching factor on system performance is investigated. We consider both AF and DF protocols in our analysis.

\subsection{Amplify-and-Forward Protocol}

Let $x_{\mathrm{u}}$ denote the transmitted signal from the user during the second phase and $P_{\mathrm{u}}$ denote the power of this signal. The received signal at $\mathrm{R}$ during this phase is espressed as

$$
y_{\mathrm{r}}=\sqrt{P_{\mathrm{u}}} g x_{\mathrm{u}}+n_{\mathrm{r}}
$$

where $n_{\mathrm{r}} \sim N\left(0, N_{0}\right)$ denotes the Gaussian distributed noise at the relay $\mathrm{R}$. 
During the third phase of transmission, the relay amplifies the received signal from mobile user and forwards it to both the AP and the user. While the AP receives this signal for the purpose of getting information message, the user receives the same signal for energy harvesting purpose. The received signal at the AP during this phase is written as

$$
y_{\mathrm{d}}=\sqrt{P_{\mathrm{r}}} h x_{\mathrm{r}}+n_{\mathrm{d}}
$$

where $x_{\mathrm{r}}$ is the signal transmitted by the relay, which has the power of $P_{\mathrm{r}}$, and $n_{\mathrm{d}}$ is the zero-mean Gaussian noise at the AP with variance $N_{0}$. Because the transmit power of the relay comes from the energy supplied by the AP in the first phase, we must have [11]

$$
P_{\mathrm{r}}=\frac{E_{\mathrm{r}}}{(1-\alpha) T / 2}=\frac{2 \alpha \eta P_{\mathrm{ap}}|h|^{2}}{1-\alpha}=k \eta P_{\mathrm{ap}}|h|^{2}
$$

where $k=\frac{2 \alpha}{1-\alpha}$.

The signal transmitted by the relay is an amplified version of $y_{\mathrm{r}}$ :

$$
x_{\mathrm{r}}=\sqrt{\beta} y_{\mathrm{r}} .
$$

According to energy conservation law, the energy consumed by the relay cannot exceed its available energy, which yields [11]

$$
\beta=\frac{1}{P_{\mathrm{u}}|g|^{2}+N_{0}} .
$$

Now, we can substitute (1), (4), and (5) into (2) and get

$$
\begin{aligned}
y_{\mathrm{d}} & =\sqrt{P_{\mathrm{r}}} h \sqrt{\beta}\left(\sqrt{P_{\mathrm{u}}} g x_{\mathrm{u}}+n_{\mathrm{r}}\right)+n_{\mathrm{d}} \\
& =\underbrace{\sqrt{P_{\mathrm{r}}} h \sqrt{\beta} \sqrt{P_{\mathrm{u}}} g x_{\mathrm{u}}}_{\text {Signal part }}+\underbrace{\sqrt{P_{\mathrm{r}}} h \sqrt{\beta} n_{\mathrm{r}}+n_{\mathrm{d}}}_{\text {Noise part }} .
\end{aligned}
$$

From (6), the signal-to-noise ratio at the AP can be computed by

$$
S N R=\frac{P_{\mathrm{r}} \beta P_{\mathrm{u}}|h|^{2}|g|^{2}}{P_{\mathrm{r}} \beta|h|^{2} N_{0}+N_{0}}=\frac{P_{\mathrm{u}}|h|^{2}|g|^{2}}{N_{0}\left(|h|^{2}+\frac{P_{\mathrm{u}}|g|^{2}+N_{0}}{k \eta P_{\mathrm{ap}}|h|^{2}}\right.} .
$$

Let's move on to determine $P_{\mathrm{u}}$. We know that the received signal at the mobile user during the third transmission phase is $y_{\mathrm{u}}=g x_{\mathrm{r}}=g \sqrt{\beta} y_{\mathrm{r}}$. Hence, the energy harvested during this phase can be determined by

$$
\begin{aligned}
E_{\mathrm{u}} & =\eta|g|^{2} \beta \mathrm{E}\left\{\left|y_{\mathrm{r}}\right|^{2}\right\}(1-\alpha) T / 2 \\
& =\eta|g|^{2} E_{\mathrm{r}}=\eta^{2} P_{\mathrm{ap}}|h|^{2}|g|^{2} \alpha T .
\end{aligned}
$$

So, the transmit power of the mobile user during the second phase is expressed as

$$
P_{\mathrm{u}}=\frac{E_{\mathrm{u}}}{(1-\alpha) T / 2}=k \eta^{2} P_{\mathrm{ap}}|h|^{2}|g|^{2} .
$$

By substituting (9) into (7) and doing some algebra, we obtain the overall SNR for AF protocol:

$$
S N R_{\mathrm{AF}}=\frac{k \eta^{2} P_{\mathrm{ap}}|h|^{4}|g|^{4}}{|h|^{2} N_{0}+\eta N_{0}|g|^{4}+\frac{N_{0}^{2}}{k \eta P_{\mathrm{ap}}|h|^{2}}} .
$$

Assume that the source transmits at a constant rate $R$, then $\gamma=2^{R}-1$ is the lower threshold for SNR. Here, the outage probability $P_{\text {out }}$ and the average throughput of the system can be evaluated by [6]

$$
\begin{gathered}
P_{\text {out }, \mathrm{AF}}=\operatorname{Pr}\{S N R<\gamma\}, \\
R_{\mathrm{AF}}=\left(1-P_{\text {out }, \mathrm{AF}}\right) R \frac{1-\alpha}{2} .
\end{gathered}
$$

The main contribution of this paper is to derive the closed-form expression of the outage probability and average throughput of the system of interest, as well as to figure out the optimal time-switching factor for energy harvesting. The results for AF protocol are formally stated in the following theorems. Theorem 1 provides the exact integral forms for the outage probability and throughput of the proposed system with AF protocol. In Theorem 2, closed-form approximations of the outage probability and throughput in terms of Meijer function are derived for high source-power-to-noiseratio regime.

Theorem 1 (AF Protocol) For the AF protocol, the outage probability and the average throughput of the proposed system can be expressed as

$$
P_{\text {out }, \mathrm{AF}}=1-\lambda_{h} \int_{\sqrt{\frac{\gamma}{\delta}}}^{\infty} \mathrm{e}^{-\lambda_{h} x-\lambda_{g} \sqrt{\frac{\gamma \delta x^{2}+\gamma}{\eta \delta^{2} x^{3}-\eta \gamma \delta x}}} \mathrm{~d} x
$$

and

$$
R_{\mathrm{AF}}=\frac{R \lambda_{h}(1-\alpha)}{2} \int_{\sqrt{\frac{\gamma}{\delta}}}^{\infty} \mathrm{e}^{-\lambda_{h} x-\lambda_{g} \sqrt{\frac{\gamma \delta x^{2}+\gamma}{\eta \delta^{2} x^{3}-\eta \gamma \delta x}}} \mathrm{~d} x
$$

where $\delta=k \eta \frac{P_{\mathrm{ap}}}{N_{0}}$.

Proof 1 See Appendix A.

Theorem 2 (AF Protocol - Closed-form approximation) At high $P_{\mathrm{ap}} / N_{0}$ regime, the outage probability and average throughput of the proposed system with AF protocol can be respectively approximated to

$$
P_{\text {out }, \mathrm{AF}} \approx 1-\frac{\mathrm{e}^{-\lambda_{h} \sqrt{\frac{\gamma}{\delta}}}}{\sqrt{\pi}} \cdot G_{0,3}^{3,0}\left(\frac{\lambda_{g}^{2} \gamma \lambda_{h}}{4 \eta \delta} \mid 0, \frac{1}{2}, 1\right)
$$

and

$$
R_{\mathrm{AF}} \approx \frac{R(1-\alpha) \mathrm{e}^{-\lambda_{h} \sqrt{\frac{\gamma}{\delta}}}}{2 \sqrt{\pi}} \cdot G_{0,3}^{3,0}\left(\frac{\lambda_{g}^{2} \gamma \lambda_{h}}{4 \eta \delta} \mid 0, \frac{1}{2}, 1\right)
$$

where $G_{p, q}^{m, n}(\cdot \mid \cdots)$ is the Meijer function (Sec. 9.3 of [13]).

Proof 2 See Appendix B. 


\subsection{Decode-and-Forward Protocol}

For DF relaying protocol, the data communication is divided into two separating hops, which do not depend on each other. Hence, the outage occurs if and only if either the source-relay path or the relay-destination path fails to satisfy the corresponding SNR constraint. Different from the AF protocol, the message transmitted by the relay during the third transmission phase is the decoded message $\hat{x}_{\mathrm{r}}$, instead of $x_{\mathrm{r}}$, and the transmit power of the relay in this phase is the same as the one given in (3). Hence, the energy harvested by the mobile user during the same transmission phase is $E_{\mathrm{u}}=\eta|g|^{2} P_{\mathrm{r}} \frac{(1-\alpha) T}{2}=\eta^{2} P_{\mathrm{ap}}|h|^{2}|g|^{2} \alpha T$. As a result, the transmit power of the mobile user in the second phase is the same as in (9).

According to the equations (1) and (2), the SNR values at the relay $\mathrm{R}$ and the $\mathrm{AP}$ are respectively determined by

and

$$
\begin{gathered}
S N R_{\mathrm{R}}=\frac{P_{\mathrm{u}}|g|^{2}}{N_{0}}=\delta \eta|h|^{2}|g|^{4} \\
S N R_{\mathrm{AP}}=\frac{P_{\mathrm{r}}|h|^{2}}{N_{0}}=\delta|h|^{4} .
\end{gathered}
$$

The outage probability of the system can be written as

$$
\begin{aligned}
P_{\text {out }, \mathrm{DF}} & =\operatorname{Pr}\left(\min \left\{S N R_{\mathrm{R}}, S N R_{\mathrm{AP}}\right\}<\gamma\right) \\
& =1-\operatorname{Pr}\left(S N R_{\mathrm{R}} \geq \gamma, S N R_{\mathrm{AP}} \geq \gamma\right) .
\end{aligned}
$$

Now we can claim the following theorem on the outage probability and the average throughput of the system of interest.

Theorem 3 (DF Protocol) For the DF protocol, the outage probability and the average throughput of the proposed system can be expressed as

$$
P_{\mathrm{out}, \mathrm{DF}}=1-\Gamma\left(1, \lambda_{h} x_{0} ; \lambda_{g} y_{0} \sqrt{x_{0} \lambda_{h}}, \frac{1}{2}\right)
$$

and

$$
R_{\mathrm{DF}}=\frac{R(1-\alpha)}{2} \Gamma\left(1, \lambda_{h} x_{0} ; \lambda_{g} y_{0} \sqrt{x_{0} \lambda_{h}}, \frac{1}{2}\right)
$$

where $\Gamma(\alpha, x ; b, \beta) \triangleq \int_{x}^{\infty} t^{\alpha-1} \mathrm{e}^{-t-b t^{-\beta}} \mathrm{d} t$ is the extended incomplete gamma function, which is defined in [14], and $x_{0}, y_{0}$ are defined by

$$
\begin{gathered}
x_{0}=\sqrt{\frac{\gamma N_{0}}{k \eta P_{\mathrm{ap}}}}, \\
y_{0}=\sqrt{\frac{\gamma N_{0}}{k \eta^{2} P_{\mathrm{ap}} x_{0}}} .
\end{gathered}
$$

Proof 3 See Appendix $C$.

\subsection{Optimal Time-Switching}

To find the optimal time-switching factors that give the best performance in terms of outage probability or average throughput, we solve the equations $\frac{\mathrm{d} P_{\text {out }}(\alpha)}{\mathrm{d} \alpha}=0$ and $\frac{\mathrm{d} R(\alpha)}{\mathrm{d} \alpha}=0$, respectively, where $P_{\text {out }}(\alpha)$ and $R(\alpha)$ are outage probability and throughput functions with respect to the time-switching factor.

By investigating the outage probability functions with respect to $\alpha$ for both $\mathrm{AF}$ and $\mathrm{DF}$, we can easily see that these are non-increasing functions. That means, the best outage performance is obtained when we exploit energy harvesting at full-scale. However, we should keep in mind that this outage performance only based on the comparison of power between signal and noise. It ignores other factors of communication process. In practice, we cannot set $\alpha$ to 1 because it means that no communication data is transferred.

Hence, the average throughput should be a more reasonable performance factor to be optimized. By plotting the throughput functions for AF and DF protocols versus $\alpha$, we learn that these functions are concave functions, which have a unique maxima on the interval $[0,1]$. The optimal factor $\alpha^{*}$ can be found numerically by some iterative methods, for instance, Golden section search method [15].

\section{Numerical Results and Discussion}

In this section, we conduct Monte Carlo simulation to verify the analysis developed in the previous section. For simplicity, in our simulation model, we assume that the sourcerelay and relay-destination distances are both normalized to unit value. Other simulation parameters are listed in Tab. 1.

\begin{tabular}{|c|c|c|}
\hline Symbol & Name & Values \\
\hline$R$ & Source rate & $3 \mathrm{bps} / \mathrm{Hz}$ \\
$\gamma$ & SNR threshold & 7 \\
$\eta$ & Energy harvesting efficiency & 0.9 \\
$\lambda_{h}$ & Mean of $|h|^{2}$ & 0.5 \\
$\lambda_{g}$ & Mean of $|g|^{2}$ & 0.5 \\
$P_{\text {ap }} / N_{0}$ & Source (AP) Power to Noise Ratio & $0-20 \mathrm{~dB}$ \\
\hline
\end{tabular}

Tab. 1. Simulation parameters.

\subsection{Amplify-and-Forward Protocol}

In Figures 3 and 4, the achievable throughput and outage probability of the system with AF protocol are plotted against $P_{\text {ap }} / N_{0}$ ratio with the data rate set to be $3 \mathrm{bps}$. The time-switching factor $\alpha$ is chosen to be 0.3 and 0.7 . It's can be observed that the outage probability is a decreasing function with respect to $P_{\text {ap }} / N_{0}$, while the throughput grows with $P_{\text {ap }} / N_{0}$. In addition, the simulation and the analysis curves are overlapping. The approximate outage probability and throughput are also plotted in these figures. They are close to the exact curves, especially when $P_{\text {ap }} / N_{0}$ is large. This confirms the correctness of our analysis in the previous section. 


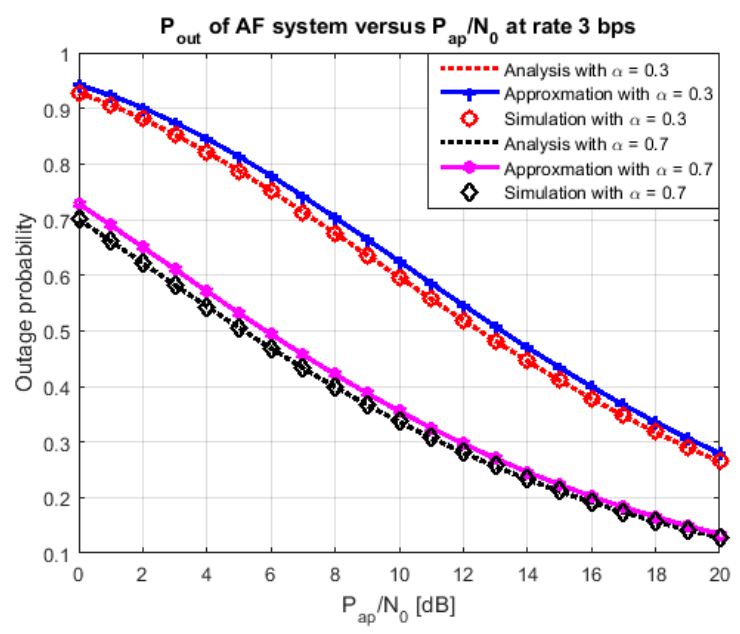

Fig. 3. Outage probability versus source power to noise ratio for AF protocol.

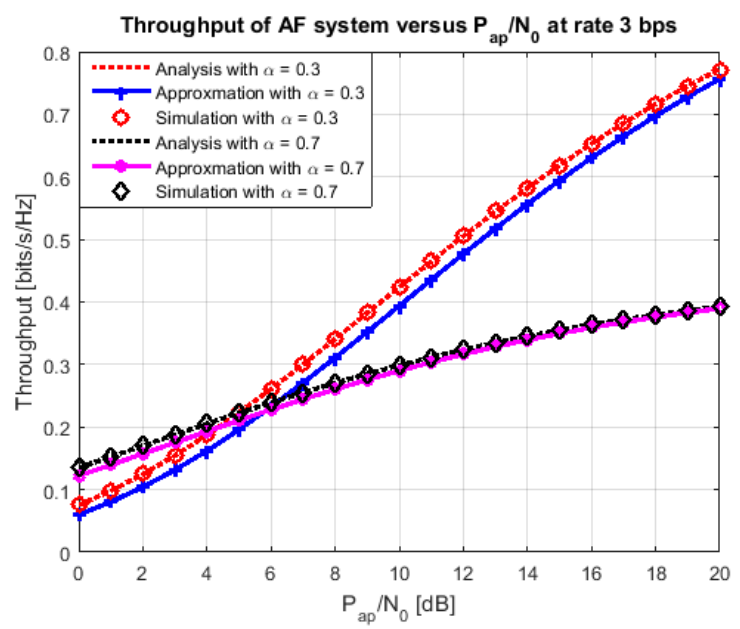

Fig. 4. Throughput versus source power to noise ratio for $\mathrm{AF}$ protocol.

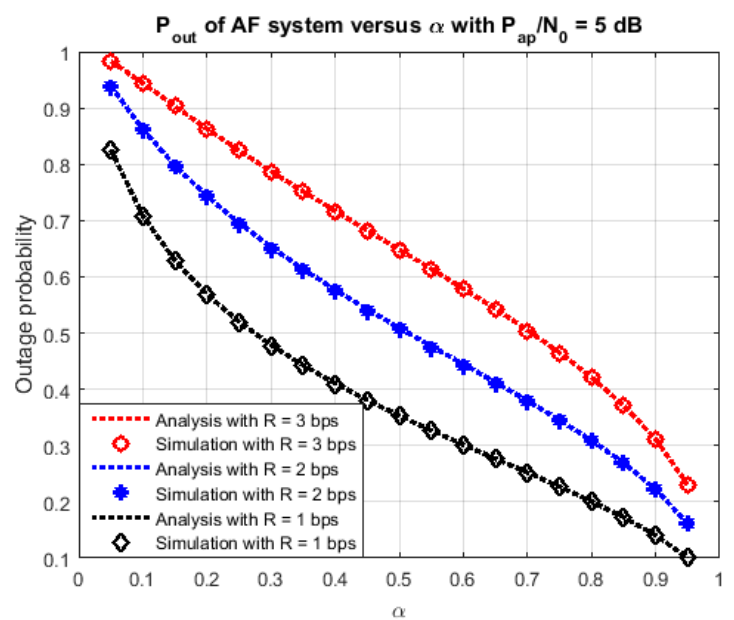

Fig. 5. Outage probability versus time-switching factor for $\mathrm{AF}$ protocol.

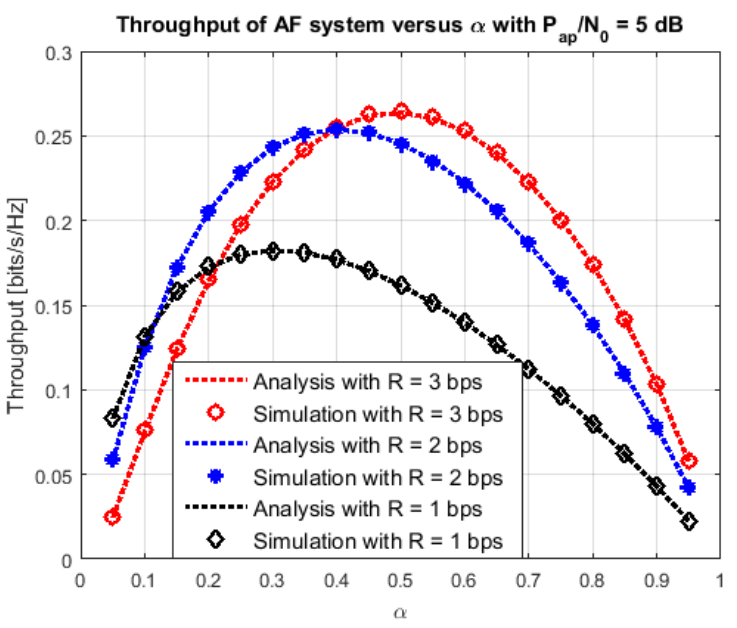

Fig. 6. Throughput versus time-switching factor for AF protocol.

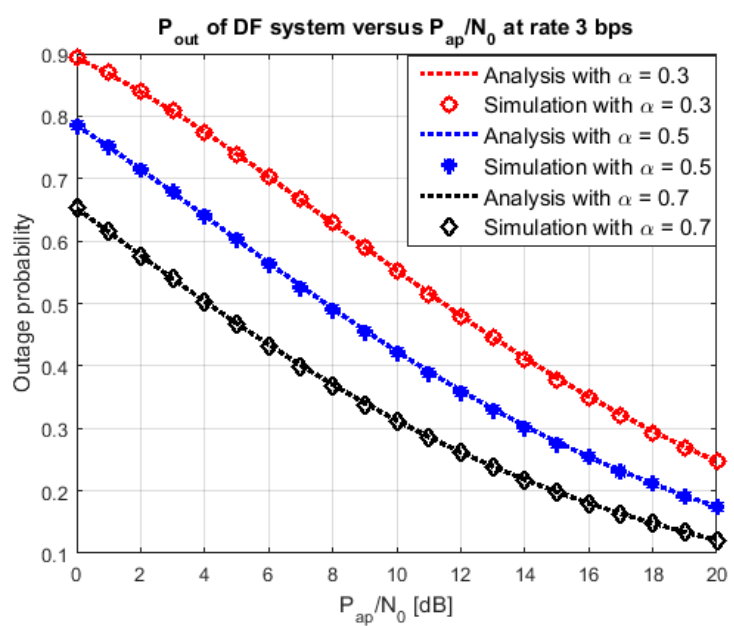

Fig. 7. Outage probability versus source power to noise ratio for DF protocol.

The impact of time switching factor on system performance with AF protocol is illustrated in Fig. 5 and 6. In this experiment, $P_{\mathrm{ap}} / N_{0}$ is set to $5 \mathrm{~dB}$, and the rate can be varied at $3 \mathrm{bps}, 2 \mathrm{bps}$, and $1 \mathrm{bps}$. It can be observed that the outage probability is reduced when we increase the value of $\alpha$. On the other hand, the simulation result shows that there exists a unique time switching factor at which the average throughput is maximized. Indeed, this optimal factor can be found iteratively using numerical methods.

\subsection{Decode-and-Forward Protocol}

For decode-and-forward protocol, we also have similar results about the impact of various parameters, such as $P_{\text {ap }} / N_{0}$ and $\alpha$ on the average throughput and the outage probability of the system. Specifically, Fig. 7 and 8 respectively plot the outage probability and throughput against $P_{\text {ap }} / N_{0}$, while Fig. 9 and 10 show the dependence of these performance characteristics on time-switching factor $\alpha$. 


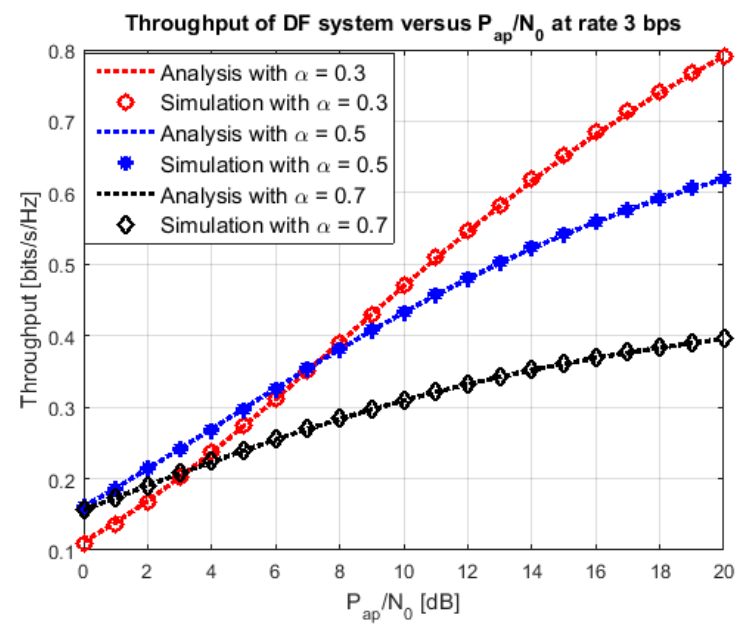

Fig. 8. Throughput versus source power to noise ratio for DF protocol

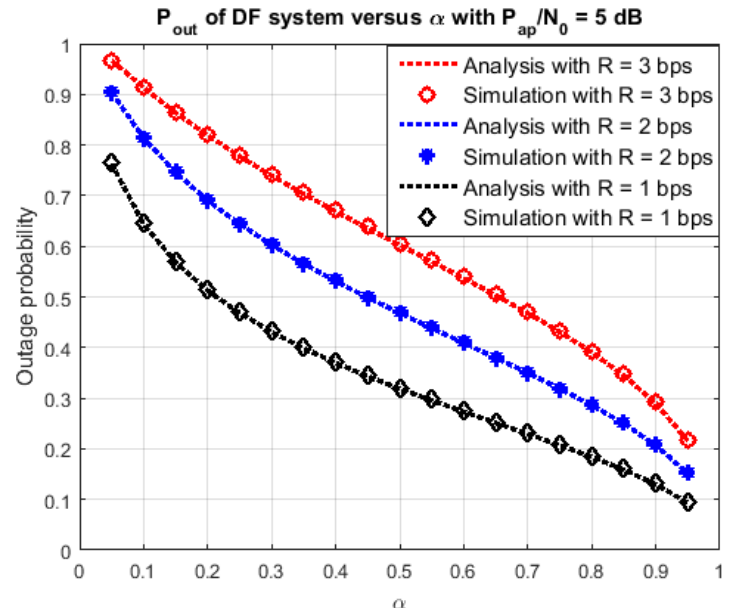

Fig. 9. Outage probability versus time-switching factor for DF protocol.

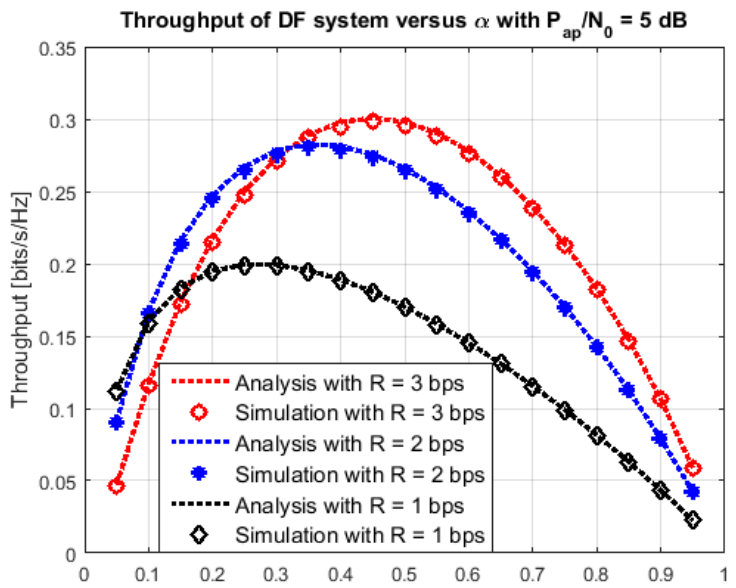

Fig. 10. Throughput versus time-switching factor for DF protocol.

\subsection{Comparison Between DF and AF}

Figures 11 and 12 compare the performance of two protocols that are considered in this paper. The results show that the DF protocol is slightly better

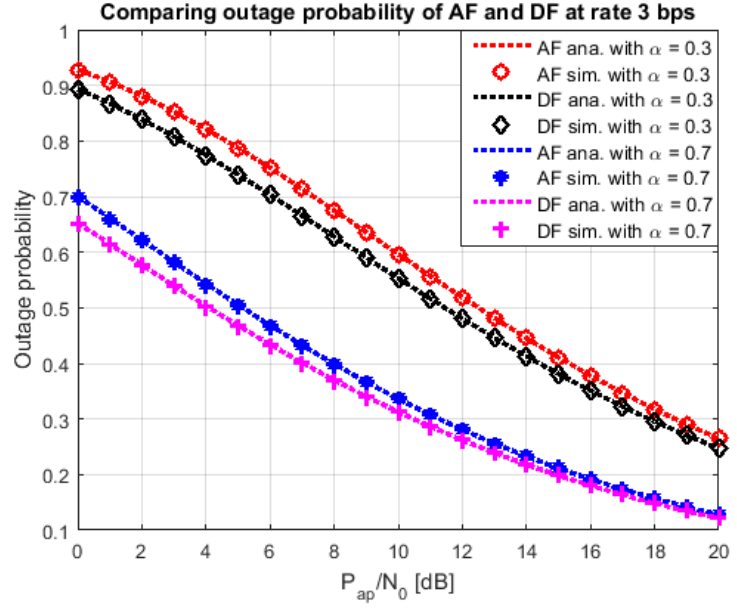

Fig. 11. Outage probability of AF and DF protocol at rate $3 \mathrm{bps}$.

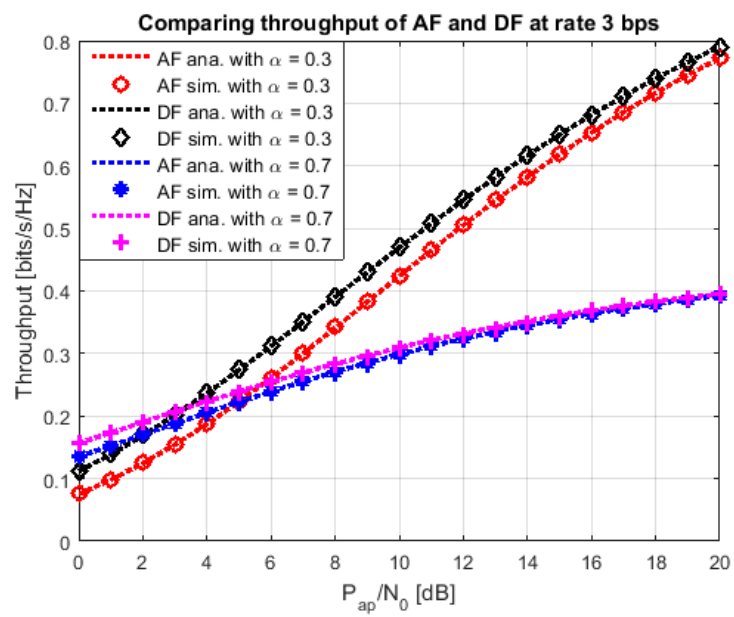

Fig. 12. Throughput of $A F$ and $D F$ protocol at rate $3 \mathrm{bps}$.

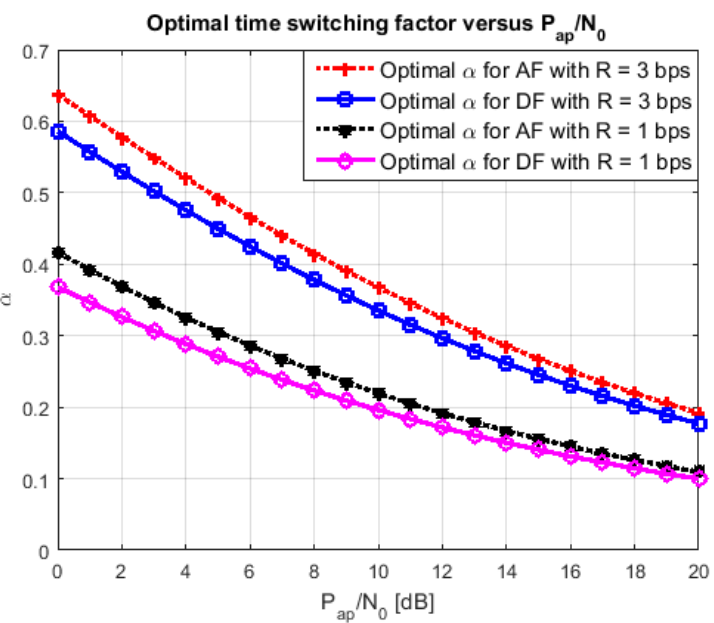

Fig. 13. Optimal time-switching factor versus $P_{\text {ap }} / N_{0}$.

than $\mathrm{AF}$ protocol in terms of both outage probability and throughput, because the noise at relay is eliminated in DF protocol, while it's accumulated and amplified in AF protocol. 


\subsection{Optimal Time-Switching Factor}

Finally, the optimal values of $\alpha$ at different values of source-power-to-noise-ratio for both $\mathrm{AF}$ and $\mathrm{DF}$ protocols are shown in Fig. 13. We can see that the $\alpha$ value that optimizes the throughput has tendency to decrease when $P_{\text {ap }} / N_{0}$ increases.

\section{Conclusions}

In this paper, we investigate the performance of a new WPC system with a bidirectional information/energy forwarding relay in the Rayleigh fading environment. Two relaying protocols based on AF and DF strategies at the relay are considered in our work. For practical orientation, we employ the time switching protocol for energy harvesting. The exact-forms of outage probability as well as the average throughput of the proposed system are derived rigorously. Numerical results are provided to verify our analysis. The results show that the outage probability decreases as the time switching factor increases, while there is a unique value of time switching factor such that the throughput is maximized. For comparison between relaying protocols, the DF protocol is slightly better than its counterpart.

While the motivation of this paper comes from the energy problem in wireless sensor networks, the analysis obtained in this work does not limit to wireless sensor networks themselves, but can be applied for a wide range of wireless applications that employ the relay-node idea. Due to this reason, some specific issues related to sensor networks have not been considered in this paper. For example, the energy required by sensor nodes when collecting data or making measurement should be taken into account. In that case, the energy source not only comes from the information source node but also from other available nodes. This harvested energy can be modeled as a randomly varying variable. That should be our further work in this topic. In addition, we can take into account other factors such as CSI error and hardware impairment.

\section{References}

[1] BI, S., HO, C. K., ZHANG, R. Wireless powered communication: opportunities and challenges. IEEE Communications Magazine, 2015, vol. 53, no. 4, p. 117-125. DOI: 10.1109/MCOM.2015.7081084

[2] ZENG, Y.ZHANG, R. Optimized training design for wireless energy transfer. IEEE Transactions on Communications, 2015, vol. 63, no. 2 , p. 536-550. DOI: 10.1109/TCOMM.2014.2385077

[3] BI, S., ZENG, Y., ZHANG, R. Wireless powered communication networks: An overview. IEEE Wireless Communications, 2016, vol. 23, no. 2, p. 10-18. DOI: 10.1109/MWC.2016.7462480

[4] HUANG, K. LAU, V. K. N. Enabling wireless power transfer in cellular networks: Architecture, modeling and deployment. IEEE Transactions on Wireless Communications, 2014, vol. 13, no. 2 , p. 902-912. DOI: 10.1109/TWC.2013.122313.130727
[5] NASIR, A. A., ZHOU, X., DURRANI, S., et al. Relaying protocols for wireless energy harvesting and information processing. IEEE Transactions on Wireless Communications, 2013, vol. 12, no. 7, p. 3622-3636. DOI: 10.1109/TWC.2013.062413.122042

[6] NGUYEN, T. N., DO, D.-T., TRAN, P. T., et al. Time switching for wireless communications with full-duplex relaying in imperfect CSI condition. KSII Transactions on Internet and Information Systems, 2016, vol. 10, no. 9, p. 4223-4239. DOI: 10.3837/tiis.2016.09.011

[7] DUY, T. T. KONG, H. Y. Outage analysis of cognitive spectrum sharing for two-way relaying schemes with opportunistic relay selection over i.n.i.d. rayleigh fading channels. IEICE Transactions on Communications, 2013, vol. E96.B, no. 1, p. 348-351. DOI: $10.1587 /$ transcom.E96.B.348

[8] SON, P. N. KONG, H. Y. Exact outage analysis of energy harvesting underlay cooperative cognitive networks. IEICE Transactions on Communications, 2015, vol. E98.B, no. 4, p. 661-672. DOI: $10.1587 /$ transcom.E98.B.661

[9] ZHANG, Y., PFLUG, H., VISSER, H. J., et al. Wirelessly powered energy autonomous sensor networks. In 2014 IEEE Wireless Communications and Networking Conference (WCNC). 2014, p. 2444-2449. DOI: 10.1109/WCNC.2014.6952732

[10] XU, J., ZHONG, Z., AI, B. Wireless powered sensor networks: Collaborative energy beamforming considering sensing and circuit power consumption. IEEE Wireless Communications Letters, 2016, vol. 5, no. 4, p. 344-347. DOI: 10.1109/LWC.2016.2558503

[11] ZENG, Y., CHEN, H., ZHANG, R. Bidirectional wireless information and power transfer with a helping relay. IEEE Communications Letters, 2016, vol. 20, no. 5, p. 862-865. DOI: 10.1109/LCOMM.2016.2549515

[12] GURAKAN, B., OZEL, O., YANG, J., et al. Energy cooperation in energy harvesting communications. IEEE Transactions on Communications, 2013, vol. 61, no. 12, p. 4884-4898. DOI: 10.1109/TCOMM.2013.110113.130184

[13] ZWILLINGER, D., MOLL, V., GRADSHTEYN, I., et al. Table of Integrals, Series, and Products. 8th ed. Boston: Academic Press, 2015. ISBN: 978-0-12-384933-5

[14] CHAUDHRY, M. A. ZUBAIR, S. M. Extended incomplete gamma functions with applications. Journal of Mathematical Analysis and Applications, 2002, vol. 274, no. 2, p. 725 - 745 . DOI: http://dx.doi.org/10.1016/S0022-247X(02)00354-2

[15] CHONG, E. K. P. ZAK, S. H., An Introduction to Optimization. 4th ed. John Wiley \& Sons, 2013. ISBN: 978-1-118-27901-4

\section{About the Authors...}

Tan N. NGUYEN was born in 1986 in Nha Trang City, Vietnam. He received B.S. and M.S. degrees in Electronics and Telecommunications Engineering from Ho Chi Minh University of Natural Sciences, Ho Chi Minh City, Vietnam in 2008 and 2012, respectively. In 2013, he joined the Faculty of Electrical and Electronics Engineering of Ton Duc Thang University, Vietnam as a lecturer. He is currently pursuing his Ph.D. degree in Electrical Engineering at VSB Technical University of Ostrava, Czech Republic. His major interests are cooperative communications, cognitive radio, and physical layer security.

Phuong T. TRAN (corresponding author) was born in 1979 in Ho Chi Minh City, Vietnam. He received B.Eng. and M.Eng degrees in Electrical Engineering from Ho Chi Minh University of Technology, Ho Chi Minh City, Vietnam in 
2002 and 2005, respectively. In 2007, he became a Vietnam Education Foundation Fellow at Purdue University, U.S.A., where he received his Ph.D. degree in Electrical and Computer Engineering in 2013. In 2013, he joined the Faculty of Electrical and Electronics Engineering of Ton Duc Thang University, Vietnam and served as the Vice Dean of Faculty since October 2014. His major interests are in the area of wireless communications and network information theory.

Hoang-Sy NGUYEN was born in Binh Duong province, Vietnam. He received the B.S. and MS.c degree from the Department of Computer Science from Ho Chi Minh City University of Information Technology (UIT-HCMC), Vietnam in 2007, 2013, respectively. He is currently pursuing the Ph.D. in School of Electrical Engineering and Computer Science, Technical University of Ostrava, Czech Republic. His research interests include energy efficient wireless communications, 5G wireless, network security, low-power networks, cloud and distributed networks.

Dinh-Thuan DO received the B.S. degree, M. Eng. degree, and Ph.D. degree from Vietnam National University (VNU-HCMC) in 2003, 2007, and 2013 respectively, all in Communications Engineering. He was a visiting Ph.D. student with Communications Engineering Institute, National Tsing Hua University, Taiwan from 2009 to 2010. Prior to joining Ton Duc Thang University in 2010, he was senior engineer at the VinaPhone Mobile Network from 2003 to 2009. Dr. Thuan was recipient of Golden Globe Award from Vietnam Ministry of Science and Technology in 2015. His research interest includes signal processing in wireless communications network, mmWave, device-to-device networks, cooperative communications, full-duplex transmission and energy harvesting.

Miroslav VOZNAK born in 1971 is an associate professor with the Department of Telecommunications, Technical University of Ostrava, Czech Republic and foreign professor with Ton Duc Thang University in Ho Chi Minh City, Vietnam. He received his Ph.D. degree in telecommunications in 2002 at the Technical University of Ostrava. He is a senior researcher in the Supercomputing center IT4Innovations in Ostrava, Czech Republic, a member of editorial boards of several journals and boards of conferences. Topics of his research interests are IP telephony, wireless networks, speech quality and network security.

\section{Appendix A: Proof of Theorem 1}

Let's denote $X=|h|^{2}, Y=|g|^{2}$, and $\delta=k \eta \frac{P_{\text {ap }}}{N_{0}}$. Note that $X$ and $Y$ are exponential random variables with the parameters $\lambda_{h}$ and $\lambda_{g}$, respectively. The end-to-end SNR of the system can be rewritten as

$$
S N R_{\mathrm{AF}}=\frac{\eta \delta X^{2} Y^{2}}{X+\eta Y^{2}+\frac{1}{\delta X}}=\frac{\eta \delta^{2} X^{3} Y^{2}}{\delta X^{2}+\eta \delta X Y^{2}+1} .
$$

By substituting (A.1) to (11), we obtain

$$
\begin{aligned}
P_{\text {out }, \mathrm{AF}} & =\operatorname{Pr}\left(\frac{X^{3} Y^{2}}{\delta X^{2}+\eta \delta X Y^{2}+1}<\frac{\gamma}{\eta \delta^{2}}\right) \\
& =\operatorname{Pr}\left\{Y^{2}\left(X^{3}-\frac{\gamma X}{\delta}\right)<\left(\frac{\gamma X^{2}}{\eta \delta}+\frac{\gamma}{\eta \delta^{2}}\right)\right\} \\
& =\operatorname{Pr}\left(X^{3}<\frac{\gamma X}{\delta}\right) \\
& +\operatorname{Pr}\left\{Y^{2}<\frac{\frac{\gamma X^{2}}{\eta \delta}+\frac{\gamma}{\eta \delta^{2}}}{X\left(X^{2}-\frac{\gamma}{\delta}\right)} \text { and } X^{3} \geqslant \frac{\gamma X}{\delta}\right\}
\end{aligned}
$$

The first term of (A.2) can be determined easily as

$$
\operatorname{Pr}\left(X^{3}<\frac{\gamma X}{b}\right)=\operatorname{Pr}\left(X<\sqrt{\frac{\gamma}{\delta}}\right)=1-\mathrm{e}^{-\lambda_{h} \sqrt{\frac{\gamma}{\delta}}}
$$

We proceed to evaluate the second term of (A.2). Let I denote this term, then we can write

$$
\begin{aligned}
I & =\operatorname{Pr}\left\{Y<\sqrt{\frac{\gamma\left(\delta X^{2}+1\right)}{\eta \delta X\left(\delta X^{2}-\gamma\right)}} \text { and } X \geqslant \sqrt{\frac{\gamma}{\delta}}\right\} \\
& =\int_{\sqrt{\frac{\gamma}{\delta}}}^{\infty} f_{X}(x) \mathrm{d} x \int_{0}^{g(x)} f_{Y}(y) \mathrm{d} y \\
& =\int_{\sqrt{\frac{\gamma}{\delta}}}^{\infty} \lambda_{h} \mathrm{e}^{-\lambda_{h} x}\left(1-\mathrm{e}^{-\lambda_{g} g(x)}\right) \mathrm{d} x \\
& =\mathrm{e}^{-\lambda_{h} \sqrt{\frac{\gamma}{\delta}}}-\int_{\sqrt{\frac{\gamma}{\delta}}}^{\infty} \lambda_{h} \mathrm{e}^{-\lambda_{h} x-\lambda_{g} g(x)} \mathrm{d} x
\end{aligned}
$$

where $g(x)=\sqrt{\frac{\gamma\left(\delta x^{2}+1\right)}{\eta \delta x\left(\delta x^{2}-\gamma\right)}} ; f_{X}(x)$ and $f_{Y}(y)$ are the pdf of $\mathrm{X}$ and $\mathrm{Y}$, respectively.

Now, (13) can be obtained by substituting (A.3) and (A.4) into (A.2). Then, we substitute (13) into (12) to get (14).

\section{Appendix B: Proof of Theorem 2}

Obviously, $\frac{1}{\delta} \rightarrow 0$ as $\frac{P_{\mathrm{ap}}}{N_{0}} \rightarrow \infty$. Hence, at high values of $\frac{P_{\mathrm{ap}}}{N_{0}}$, we can use the following approximation:

$$
\frac{X+\eta Y^{2}+\frac{1}{\delta X}}{X+\eta Y^{2}+\sqrt{\frac{\gamma}{\delta}}} \approx 1 .
$$

Now, by multiplying this term to the expression of $S N R_{\mathrm{AF}}$ in (A.1), we obtain

$$
S N R_{\mathrm{AF}}=\frac{\eta \delta X^{2} Y^{2}}{X+\eta Y^{2}+\sqrt{\frac{\gamma}{\delta}}}
$$


Using the same procedure as in Appendix A, we have

$$
\begin{aligned}
P_{\text {out }, \mathrm{AF}} & =\operatorname{Pr}\left(\frac{\eta \delta X^{2} Y^{2}}{X+\eta Y^{2}+\sqrt{\frac{\gamma}{\delta}}}<\gamma\right) \\
& =\operatorname{Pr}\left\{\eta \delta Y^{2}\left(X^{2}-\frac{\gamma}{\delta}\right)\right\}<\gamma\left(X+\sqrt{\frac{\gamma}{\delta}}\right) \\
& =\operatorname{Pr}\left(X^{2}<\frac{\gamma}{\delta}\right) \\
& +\operatorname{Pr}\left\{Y^{2}<\frac{\gamma\left(X+\sqrt{\frac{\gamma}{\delta}}\right)}{\eta \delta\left(X^{2}-\frac{\gamma}{\delta}\right)} \text { and } X^{2} \geqslant \frac{\gamma}{\delta}\right\} \\
& =1-\mathrm{e}^{-\lambda_{h} \sqrt{\frac{\gamma}{\delta}}} \\
& -\operatorname{Pr}\left\{Y^{2}<\frac{\gamma}{\eta \delta\left(X-\sqrt{\frac{\gamma}{\delta}}\right)} \text { and } X^{2} \geqslant \frac{\gamma}{\delta}\right\}
\end{aligned}
$$

Again, we use the same argument as in the proof of Theorem 1 to rewrite the last term of (B.2) as

$$
\begin{aligned}
I & =\operatorname{Pr}\left\{Y<\sqrt{\frac{\gamma}{\eta \delta\left(X-\sqrt{\frac{\gamma}{\delta}}\right)}} \text { and } X \geqslant \sqrt{\frac{\gamma}{\delta}}\right\} \\
& =\mathrm{e}^{-\lambda_{h} \sqrt{\frac{\gamma}{\delta}}}-\int_{\sqrt{\frac{\gamma}{\delta}}}^{\infty} \lambda_{h} \mathrm{e}^{-\lambda_{h} x-\lambda_{g} h(x)} \mathrm{d} x
\end{aligned}
$$

where $h(x)=\sqrt{\frac{\gamma}{\eta \delta\left(x-\sqrt{\frac{\gamma}{\delta}}\right)}} ; f_{X}(x)$ and $f_{Y}(y)$ are the pdf of $\mathrm{X}$ and $\mathrm{Y}$, respectively.

Then, (B.2) can be rewritten as

$$
P_{\text {out }, \mathrm{AF}}=1-\int_{\sqrt{\frac{\gamma}{\delta}}}^{\infty} \lambda_{h} \mathrm{e}^{-\lambda_{h} x-\lambda_{g} \sqrt{\frac{\gamma}{\eta \delta\left(x-\sqrt{\frac{\gamma}{\delta}}\right)}}} \mathrm{d} x .
$$
obtain

Finally, by changing variable $u=\lambda_{h}\left(x-\sqrt{\frac{\gamma}{\delta}}\right)$, we

$$
\begin{aligned}
P_{\text {out }, \mathrm{AF}} & =1-\int_{0}^{\infty} \mathrm{e}^{-u-\lambda_{h} \sqrt{\frac{\gamma}{\delta}}-\lambda_{g} \sqrt{\frac{\gamma \lambda_{h}}{\eta \delta u}}} \mathrm{~d} u \\
& =1-\mathrm{e}^{-\lambda_{h} \sqrt{\frac{\gamma}{\delta}}} \Gamma\left(1,0 ; \lambda_{g} \sqrt{\frac{\gamma \lambda_{h}}{\eta \delta}}, \frac{1}{2}\right) \\
& =1-\frac{\mathrm{e}^{-\lambda_{h} \sqrt{\frac{\gamma}{\delta}}}}{\sqrt{\pi}} G_{0,3}^{3,0}\left(\frac{\lambda_{g}^{2} \gamma \lambda_{h}}{4 \eta \delta} \mid 0, \frac{1}{2}, 1\right)
\end{aligned}
$$

where $\Gamma(\alpha, x ; b, \beta)$ is the extended incomplete Gamma function [14] and the last equality comes from the Corollary (3.20) of [14]. From (B.5), we can easily get the throughput formula (16).

\section{Appendix C: Proof of Theorem 3}

Again, let $X$ denote $|h|^{2}$ and $Y$ denote $|g|^{2}$. The SNR values in (17) and (18) now become

$$
\begin{aligned}
S N R_{\mathrm{R}} & =\delta \eta|h|^{2}|g|^{4}=\delta \eta X Y^{2} \\
S N R_{\mathrm{AP}} & =\delta|h|^{4}=\delta X^{2}
\end{aligned}
$$

where $\delta=k \eta \frac{P_{\mathrm{ap}}}{N_{0}}$. Also, from (21) and (23), we have $x_{0}=\sqrt{\frac{\gamma}{\delta}}$ and $y_{0}=\sqrt{\frac{\gamma}{\delta \eta x_{0}}}$.

From (19), the outage probability can be rewritten as

$$
\begin{aligned}
& P_{\text {out,DF }}=1-\operatorname{Pr}\left\{\delta \eta X Y^{2} \geq \gamma, \delta X^{2} \geq \gamma\right\} \\
& =1-\operatorname{Pr}\left\{Y \geq \sqrt{\frac{\gamma}{\eta \delta X}}, X \geq x_{0}\right\} \\
& =1-\operatorname{Pr}\left\{Y \geq y_{0} \sqrt{\frac{x_{0}}{X}}, X \geq x_{0}\right\} \\
& =1-\operatorname{Pr}\left\{X \geq x_{0}, y_{0} \sqrt{\frac{x_{0}}{X}} \leq Y<y_{0}\right\} \\
& -\operatorname{Pr}\left\{X \geq x_{0}, Y \geq y_{0}\right\} \\
& =1-\mathrm{e}^{-\lambda_{h} x_{0}-\lambda_{g} y_{0}} \\
& -\int_{x_{0}}^{\infty} f_{X}(x)\left(\int_{y_{0} \sqrt{\frac{x_{0}}{x}}}^{y_{0}} f_{Y}(y) \mathrm{d} y\right) \mathrm{d} x .
\end{aligned}
$$

Denote the last term in (C.1) as $I_{1}$, then we have

$$
\begin{aligned}
I_{1} & =-\int_{x_{0}}^{\infty} \lambda_{h} \mathrm{e}^{-\lambda_{h} x}\left(\int_{y_{0} \sqrt{\frac{x_{0}}{x}}}^{y_{0}} \lambda_{g} \mathrm{e}^{-\lambda_{g} y} \mathrm{~d} y\right) \mathrm{d} x \\
& =-\int_{x_{0}}^{\infty} \lambda_{h} \mathrm{e}^{-\lambda_{h} x}\left(\mathrm{e}^{-\lambda_{g} y_{0} \sqrt{\frac{x_{0}}{x}}}-\mathrm{e}^{-\lambda_{g} y_{0}}\right) \mathrm{d} x \\
& =-\int_{x_{0}}^{\infty} \lambda_{h} \mathrm{e}^{-\lambda_{h} x} \mathrm{e}^{-\lambda_{g} y_{0} \sqrt{\frac{x_{0}}{x}}} \mathrm{~d} x+\mathrm{e}^{-\lambda_{g} y_{0}-\lambda_{h} x_{0}} \\
& =-\int_{\lambda_{h} x_{0}}^{\infty} \mathrm{e}^{-u} \cdot \mathrm{e}^{-\lambda_{g} y_{0} \sqrt{\frac{\lambda_{h} x_{0}}{u}}} \mathrm{~d} u+\mathrm{e}^{-\lambda_{g} y_{0}-\lambda_{h} x_{0}}
\end{aligned}
$$

By substituting this into (C.1) and using the definition of extended incomplete gamma function (formula (1.9) in [14]) we obtain (20). Finally, (21) is obtained by including (20) in the definitive formula of average throughput. 